\title{
Composição mineral do gafanhoto aposemático semiaquático Tetrataenia surinama (Linnaeus, 1764) (Orthoptera: Acrididae)
}

O gafanhoto aposemático Tetrataenia surinama (Linnaeus, 1764) (Orthoptera, Acrididae, Leptysminae), se alimenta e desenvolve seu ciclo de vida associado à macrófita aquática Montrichardia linifera (Arruda) Schott, (Araceae), planta conhecida como Aninga, considerada tóxica para vertebrados e que se encontra amplamente distribuída na região Amazônica, inclusive nos centros urbanos. Este trabalho teve como objetivo determinar, por meio de análise química, a composição mineral do gafanhoto T. surinama e relacioná-la com a composição mineral de sua planta hospedeira M. linifera (Aninga). A coleta dos gafanhotos e da Aninga foi feita na área da Universidade Federal Rural da Amazônia (UFRA), Belém, Pará, no período de maio a julho/2017. Os exemplares coletados foram transferidos para o Insetário da Universidade do Estado do Pará (UEPA), onde receberam dieta exclusiva com folhas de Aninga por 21 dias maio a julho/2017. Os exemplares coletados foram transferidos para o Insetário da Universidade do Estado do Pará (UEPA), onde receberam dieta exclusiva com folhas de Aninga por 21 dias
(NUNES, 1989). A análise química, para determinação da composição mineral do gafanhoto foi realizada no laboratório de análises químicas da Coordenação de Ciências da Terra e Ecologia (CCTE), do Museu Paraense Emílio Goeldi (MPEG). As análises químicas do gafanhoto (em triplicata) seguiram o protocolo de Amarante et al. (2009), que já foi empregado na análise química da Aninga. Os resultados obtidos para o gafanhoto deram positivos para Cálcio (Ca), Magnésio (Mg), Ferro (Fe), Cobre (Cu), Zinco (Zn), e Manganês (Mn). O elemento de maior concentração em T. surinama foi o Cálcio para os machos $(668,44 \mathrm{mg} / \mathrm{kg}$ ), fêmeas $(559,18 \mathrm{mg} / \mathrm{kg}$ ) e imaturos (ninfas) $(819,85 \mathrm{mg} / \mathrm{kg}$ ) e a menor concentração nos machos foi de Manganês (44,43 mg/kg) enquanto que nas fêmeas e ninfas foi de Cobre (43,26 mg/kg e 48,44 mg/kg, respectivamente). Na Aninga, o elemento de maior concentração foi o Manganês (3612,23 mg/kg), que a torn uma 'planta tóxica'. Embora T. surinama se alimente de Aninga (sua planta hospedeira), ele possui baixa concentração de Manganês e, por isso, não pode ser considerado tóxico, caso seja ingerido por consumidores na cadeia alimentar. Portanto, as 'cores de advertência' (aposematismo) existente no gafanhoto, não indica toxicidade, mas pode representar uma estratégia de sobrevivência, produto do processo de coevolução de T. surinama com sua planta hospedeira M. linifera.

Palavras-chave: Interação inseto-planta; Composição química; Toxicidade.

\section{Mineral composition of the semiaquatic aposematic grasshopper Tetrataenia surinama (Linnaeus, 1764) (Orthoptera: Acrididae)}

\begin{abstract}
The grasshopper Tetrataenia surinama (Linnaeus, 1764) (Orthoptera, Acrididae,Leptysminae), develops its life cycle associated with aquatic macrophytes Montrichardia linifera (Arruda) Schott, 1854 (Araceae) (Aninga), which is considered toxic and is extensively found in the Amazon region, including the urban centers. The objective of this papper was to determine, through chemical analysis, the mineral composition of the grasshopper T. Surinama and relate it to the mineral composition of your host plant M. linifera (Aninga). The grasshoppers and Aninga were chemical analysis, the mineral composition of the grasshopper T. Surinama and relate it to the mineral composition of your host plant M. linifera (Aninga). The grasshoppers and Aninga were
collected in Universidade Federal Rural da Amazônia (UFRA), Belém, Pará, for the period from May 27 until July 2 . The collected specimens were brought to insect breeding laboratoy of UEPA, where they received an exclusive diet with Aninga leaves for 21 days, methodology proposed by Nunes (1989). The chemical analysis, to determine the mineral composition of the grasshopper, were performed at the laboratory of chemical analyzes at Coordenação de Ciências da Terra e Ecologia (CCTE) from Museu Paraense Emílio Goeldi (MPEG). The methodology of the chemical analysis was according to the protocol proposed by Amarante et al. (2009). The results of the chemical analyzes carried out in triplicates of grasshoppers showed positive for Calcium (Ca), Magnesium (Mg), Iron (Fe), Copper ( $\mathrm{Cu})$, Zinc (Zn), Manganese (Mn). The element of highest concentration on grasshoppers was Calcium for male (668,44 mg/kg), females $(559,18 \mathrm{mg} / \mathrm{kg})$, immature $(\mathrm{nymph})(819,85 \mathrm{mg} / \mathrm{kg})$, and the lowest concentration for male was Manganese $(44,43 \mathrm{mg} / \mathrm{kg})$ whereas for females and nymphs was Copper $(43,26 \mathrm{mg} / \mathrm{kg}$ $48,44 \mathrm{mg} / \mathrm{kg}$ res 48,4 (aposematism) existing in T. surinama, can represent a survival strategy from this grasshopper species, it's the result of T. surinamas coevolutionary process with its host plant M. linifera.

Keywords: Insect-plant interection; Chemical composition; Toxicity.
\end{abstract}

Topic: Fundamentos de Biodiversidade

Reviewed anonymously in the process of blind peer.
Received: 02/07/2021

Approved: 28/07/2021
Wilson Figueiredo de Lima

Universidade do Estado do Pará, Brasil

http://lattes.cnpq.br/4951333082137719

limawilson01@gmail.com

Ana Lúcia Nunes Gutjahr (iD)

Universidade do Estado do Pará, Brasil

http://lattes.cnpq.br/0991074824474678

http://orcid.org/0000-0001-7806-3069

algutjahr@uepa.br

Carlos Elias de Souza Braga

Universidade do Estado do Pará, Brasil

http://lattes.cnpq.br/3935270777925808

http://orcid.org/0000-0002-4621-8932

carlosbraga@uepa.br

d

DOI: 10.6008/CBPC2179-6858.2021.007.0013
Cristine Bastos do Amarante (iD)

Museu Paraense Emílio Goeldi, Brasil

http://lattes.cnpq.br/4101983776191966

http://orcid.org/0000-0002-8602-8180

cbamarante@museu-goeldi.br

José Paulo Sarmento

Museu Paraense Emílio Goeldi, Brasil

http://lattes.cnpq.br/8637153534886764

sarmento@museu-goeldi.br
Referencing this:

LIMA, W. F.; GUTJAHR, A. L. N.; BRAGA, C. E. S.; AMARANTE, C. B.; SARMENTO, J. P.. Composição mineral do gafanhoto aposemático semiaquático Tetrataenia surinama (Linnaeus, 1764) (Orthoptera: Acrididae). Revista Ibero Americana de Ciências Ambientais, v.12, n.7, p.135-147, 2021. DOI: http://doi.org/10.6008/CBPC21796858.2021 .007 .0013 


\section{INTRODUÇÃO}

Atualmente a Classe Insecta representa mais de $60 \%$ das espécies conhecidas de animais, sendo, o maior grupo existente atualmente (RAFAEL et al., 2012). Devido à grande abundância, nos mais diferentes ecossistemas, os insetos são importantes ao meio ambiente, por desenvolverem funções ecológicas fundamentais, como por exemplo, a polinização de plantas e por fazerem parte das cadeias tróficas ecossistêmicas.

$\mathrm{Na}$ Classe Insecta se destaca a Ordem Orthoptera, cujos representantes são conhecidos popularmente como grilos, esperanças, paquinhas, manés-magro e gafanhotos. Um representante desta ordem é o gafanhoto amazônico Tetrataenia surinama que pertence à família Acrididae, subfamília Leptysminae e tribo Tetrataeniini (COSTA et al., 1998; NUNES-GUTJAHR et al., 2011). Esta espécie possui porte pequeno (machos $=27 \mathrm{~mm}$ e Fêmeas $=29 \mathrm{~mm}$ ) (NUNES-GUTJAHR et al., 2011) e coloração aposemática (cores de advertência), com padrão cromático, de linhas amarelas nas laterais, manchas pretas na região dorsal da cabeça e tórax e manchas vermelhas e amarelas nas pernas. T. Surinama é semiaquático e pode ser encontrado em ambientes aquáticos, semiaquáticos, alagados ou úmidos onde normalmente se alimentam da macrófita aquática, Montrichardia linifera (Araceae), que é conhecida popularmente como Aninga (AMARANTE et al., 2010).

Devido ao seu modo de vida, essa espécie de gafanhoto é comumente visualizada nos aningais de rios, lagos e outros corpos d'água amazônicos como igarapés, igapós e córregos de várzea (NUNES-GUTJAHR et al., 2011), onde a Aninga, sua planta hospedeira, é amplamente distribuída. Ressalta-se ainda, que está planta é também encontrada em cursos d'água no meio urbano.

Tanto o gafanhoto $T$. surinama quanto sua planta hospedeira foram pouco estudados, podendo se destacar os trabalhos de Costa et al. (1998) que tratou de aspectos biológicos do gafanhoto e o estudo de Amarante et al. (2011), que abordou a composição mineral da Aninga, quando foi avaliado os teores de Cálcio, Magnésio, Ferro, Cobre, Zinco e Manganês, sendo este último mineral registrado em grande concentração, que classifica está planta como tóxica para animais que dela se alimentam.

Estudos sobre a composição química de gafanhotos, até o momento são inexistentes, sendo em literatura relatada apenas a composição química de outros insetos, com ênfase em metais pesados em besouros (JELASKA et al., 2007), e alguns estudos voltados a composição nutricional, visando a entomofagia. A relação existente entre gafanhotos e sua planta hospedeira foi relatada em estudos realizados na Amazônia Central, para outras espécies como, por exemplo, Cornops aquaticum (Bruner, 1906) (Acrididae, Leptysminae) e Eichhornia crassipes (Pontedereaceae) (BRAGA, 2008). Entretanto, a composição química de plantas e de seus animais hóspedes, considerando os insetos, ainda não está registrada em literatura pertinente, embora haja estudos isolados, principalmente para o uso em cosmetologia (gorduras, óleos e essências), etnoentomologia (uso medicinal) (COSTA NETO et al., 2004; PILLA et al., 2006; RUIVO, 2012) ou referentes à criação de animais de grande porte, onde é avaliada composição química da pastagem (PACIULLO et al., 2008; RODRIGUES et al., 2010). 
Este trabalho teve como objetivo determinar, por meio de análise química a composição mineral do gafanhoto $T$. surinama e relacioná-la com a composição mineral de sua planta hospedeira $M$. linifera (Aninga), a fim de verificar se existem metais em concentrações de toxicidade, que justifique o aposematismo dessa espécie de gafanhoto semiaquático.

\section{MATERIAIS E MÉTODOS}

\section{Coleta de Gafanhotos e preparo para análises}

Para a realização das análises químicas, exemplares de $T$. surinama foram coletados no campus da Universidade Federal Rural da Amazônia (UFRA), na localidade conhecida como Estrada da Várzeam, em um aningal que sofre influência direta das marés do Rio Guamá $\left(1^{\circ} 27^{\prime} 42^{\prime \prime} \mathrm{S}, 48^{\circ} 26^{\prime} 10.2^{\prime \prime} \mathrm{W}\right)$, Belém, Pará.

As coletas de campo ocorreram no período de maio a julho/2017 no horário de 08h30min às $12 \mathrm{~h} 00 \mathrm{~min}$, utilizando rede entomológica para a captura dos gafanhotos. Após coletados, os exemplares de T. surinama foram transferidos para o insetário da Universidade do Estado do Pará (UEPA) no Campus I Centro de Ciências Sociais e Educação (CCSE), onde foram separados por estágio (adultos e ninfas) e ficaram acondicionados em béqueres de $1 \mathrm{~L}$ e aquários $(40 \times 30 \times 20 \mathrm{~cm})$ contendo água e folhas de $M$. linifera, que lhes serviram de alimento e substrato. Ressalta-se que as folhas de Aninga foram coletadas também na UFRA e, no laboratório, ficaram acondicionadas em sacos plásticos em refrigerador. Em dias alternados, era realizada a limpeza dos béqueres, dos aquários e reposição de folhas de Aninga. A temperatura no insetário variou de $25 \circ$ a $30 \circ \mathrm{C}$.

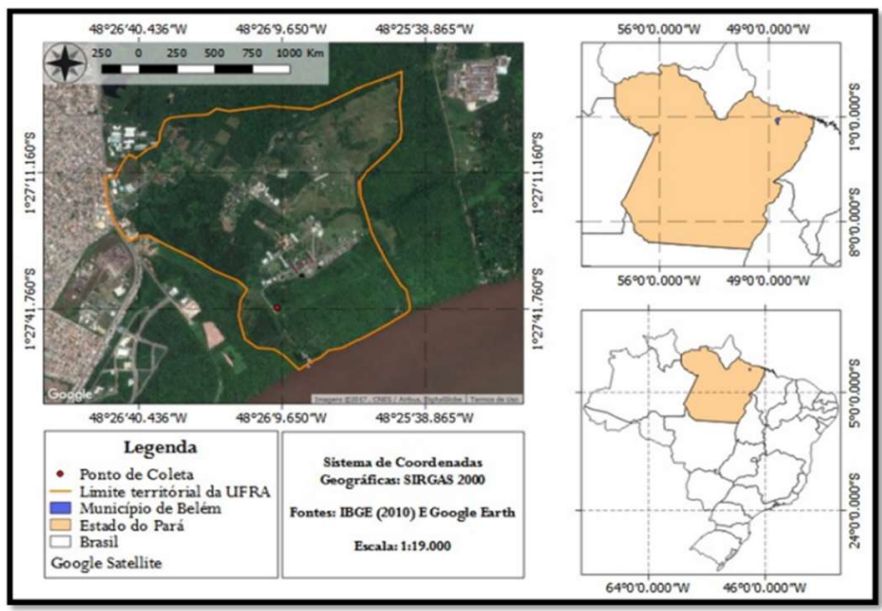

Figura 1: Localização da área e o ponto de coleta no campus da UFRA, Belém, Pará.

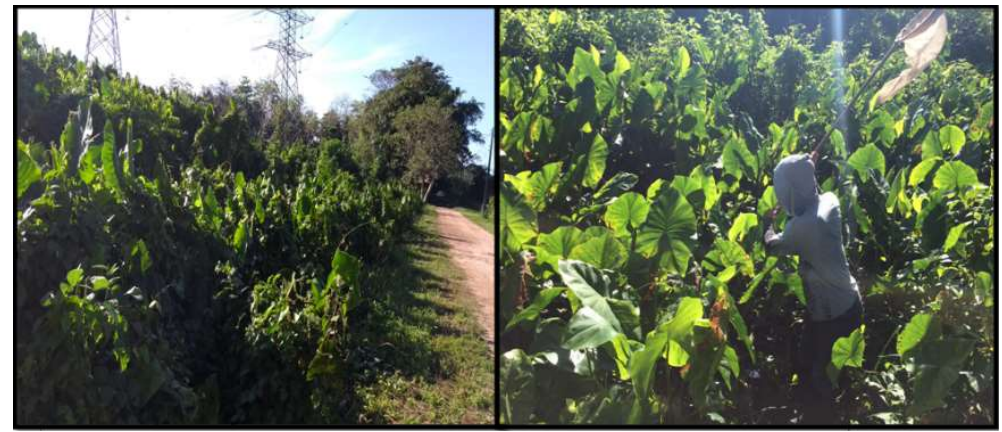

Figura 2: Aspecto do aningal na Estrada da Várzea na UFRA, onde Tetrataenia surinama foi coletado. 
No insetário, os exemplares de $T$. surinama foram alimentados por 21 dias somente com folhas de M. linifera (Aninga), conforme metodologia de Nunes (1989). Posteriormente, cada gafanhoto, foi depositado em Eppendorf (3,5 ml), anestesiados em refrigerador e sacrificados em freezer.

As análises químicas dos gafanhotos foram realizadas no Laboratório de Análises Químicas da Coordenação de Ciências da Terra e Ecologia (CCTE), do Museu Paraense Emílio Goeldi (MPEG).

\section{Procedimento para a Análise Química}

Inicialmente foi obtida a massa úmida (g) de $T$. surinama, quando os gafanhotos foram pesados em balança analítica (BEL M2202), separados por estágios, sendo utilizados no total 151 espécimes (59 machos, 51 fêmeas e 41 ninfas). Após a pesagem, os mesmos foram introduzidos em estufa a 60 을 , onde permaneceram por quatro dias até a secagem completa, sendo posteriormente retirados da estufa, resfriados em temperatura ambiente e pesados novamente, para obtenção do peso seco (g). Após a pesagem dos gafanhotos (peso seco), os mesmos foram pulverizados em um almofariz com pistilo, pesados e transferidos para um tubo de ensaio com tampa.

Para a determinação dos minerais dos gafanhotos, com o material pulverizado, foram preparadas soluções em triplicada para cada estágio dos mesmos (adultos e imaturos). As amostras corresponderam a 0,3g de massa seca pulverizada de $T$. surinama que foi depositada em tubo de digestão onde foi acrescentado $1 \mathrm{~mL}$ de peróxido de hidrogênio $\left(\mathrm{H}_{2} \mathrm{O}_{2}\right) 30 \%(\mathrm{v} / \mathrm{v})$ e $3 \mathrm{~mL}$ de ácido nítrico $\left(\mathrm{HNO}_{3}\right)$ concentrado. As amostras foram depositadas no bloco para um balão volumétrico de $50 \mathrm{~mL}$ onde foi adicionada água deionizada, até completar $50 \mathrm{~mL}$ de solução. Para evitar contaminação das amostras, foi feita a mesma abertura nítrica para um branco sem a transferência das amostras a serem analisadas. Após o procedimento descrito, as amostras estavam prontas para a determinação dos minerais no espectrômetro de absorção atômica de chama (iCE 3000 Series Atomic Absorption Spectrometers). Ressalta-se que o protocolo para a análise química do gafanhoto seguiu Amarante et al. (2011).

Neste estudo os dados referentes à macrófita $M$. linifera são decorrentes do trabalho de Amarante et al. (2009), que utilizou o mesmo protocolo de análise, aqui aplicado para T. surinama. Com a mesma solução, utilizada para determinação da composição mineral de $T$. surinama (mesmo protocolo) e com o mesmo método de análise, foi realizada também uma análise para os metais pesados Cádmio (Cd) e Chumbo $(\mathrm{Pb})$.

\section{Análise de dados}

Os dados obtidos foram analisados descritivamente no programa computacional Microsoft Excel 2010. Também foi realizada uma análise de correlação (r), quando foi obtido o coeficiente de correlação existente entre os valores da concentração dos elementos minerais do gafanhoto e de sua planta hospedeira que pode ser classificada nos níveis moderado, forte e muito forte. 


\section{RESULTADOS}

As análises químicas realizadas no gafanhoto $T$. surinama, evidenciaram resultado positivo para os elementos Cálcio (Ca), Magnésio (Mg), Ferro (Fe), Cobre (Cu), Zinco ( $\mathrm{Zn})$, e Manganês (Mn), confirmando a presença destes elementos na constituição corpórea desse gafanhoto semiaquático. No entanto, os valores de concentração desses elementos foram variáveis nos diferentes estágios de desenvolvimento do gafanhoto. Considerando o valor total dos elementos entre machos, fêmeas e imaturos, o Cálcio foi o elemento químico que apresentou maior concentração $(\mathrm{Ca}=682,49 \mathrm{mg} / \mathrm{Kg})$ e o de menor concentração foi o Cobre (Cu= 56,74 mg/Kg) (Tabela 1$)$.

Entre o estágio de adulto, nos machos o Cálcio teve maior concentração ( $\mathrm{Ca}=668,44 \mathrm{mg} / \mathrm{Kg}$ ), enquanto o elemento de menor concentração foi o Manganês ( $\mathrm{Mn}=44,43 \mathrm{mg} / \mathrm{Kg}$ ). Nas fêmeas a maior concentração foi também de Cálcio ( $\mathrm{Ca}=559,18 \mathrm{mg} / \mathrm{Kg}$ ) e o elemento que teve menor concentração foi o Cobre $(\mathrm{Cu}=48,26 \mathrm{mg} / \mathrm{Kg})$. Nos imaturos as análises químicas evidenciaram a concentração de elementos semelhantes aos das fêmeas, diferindo apenas nos valores, ou seja, uma maior concentração também de Cálcio (Ca=819,85 mg/Kg) e a menor concentração de Cobre ( $\mathrm{Cu}=48,44 \mathrm{mg} / \mathrm{Kg})$ (Tabela 1$)$.

Ressalta-se que os valores das concentrações dos elementos, neste estudo, foram resultantes da solução de triplicatas de vários indivíduos de T. surinama, em decorrência do baixo peso (massa seca/biomassa) de um exemplar de gafanhoto, o que impossibilitou a determinação da composição mineral de cada unidade de indivíduo por estágio.

Os imaturos, em relação aos adultos, apresentaram maior concentração para os elementos Cálcio ( $\mathrm{Ca}=819,85 \mathrm{mg} / \mathrm{kg}$ ); Magnésio ( $\mathrm{Mg}=744,69 \mathrm{mg} / \mathrm{kg}$ ) e Manganês ( $\mathrm{Mn}=135,07 \mathrm{mg} / \mathrm{kg}$ ) e menor concentração para o Cobre ( $\mathrm{Cu}=43,26 \mathrm{mg} / \mathrm{kg})$ (Tabela 1$)$.

Dentre os elementos químicos encontrados na constituição corpórea de $T$. surinama, além do Cálcio, o Mangnésio teve a segunda maior concentração em todos os estágios de desenvolvimento do gafanhoto (Tabela 1).

Tabela 1: Composição mineral do gafanhoto Tetrataenia surinama e das folhas de Montrichardia linifera (*).

\begin{tabular}{llllll}
\hline Elementos Químicos & $\begin{array}{l}\text { Machos } \\
(\mathrm{mg} / \mathrm{kg})\end{array}$ & $\begin{array}{l}\text { Fêmeas } \\
(\mathrm{mg} / \mathrm{kg})\end{array}$ & $\begin{array}{l}\text { Imaturos } \\
(\mathrm{mg} / \mathrm{kg})\end{array}$ & $\begin{array}{l}\text { Média Total } \\
(\mathrm{mg} / \mathrm{kg})\end{array}$ & $\begin{array}{l}\text { Aninga } \\
(\mathrm{mg} / \mathrm{kg})(*)\end{array}$ \\
\hline Cálcio & 668,44 & 559,18 & 819,85 & 682,49 & 31538,72 \\
Magnésio & 447,96 & 284,52 & 744,69 & 492,39 & 9755,09 \\
Ferro & 118,99 & 515,60 & 394,79 & 343,13 & 58,82 \\
Cobre & 121,75 & 43,26 & 48,44 & 56,74 & 6,81 \\
Zinco & 225,02 & 280,11 & 226,71 & 243,95 & 255,9 \\
Manganês & 44,43 & 76,38 & 135,07 & 85,29 & 3612,23 \\
\hline
\end{tabular}

$\left({ }^{*}\right)$ Dados de Amarante et al. (2009).

O elemento Ferro teve a maior concentração nas fêmeas ( $\mathrm{Fe}=515,60 \mathrm{mg} / \mathrm{Kg}$ ) seguido das imaturos (ninfas) $(\mathrm{Fe}=394,79 \mathrm{mg} / \mathrm{Kg}$ ) e posteriormente nos machos ( $\mathrm{Fe}=118,99 \mathrm{mg} / \mathrm{Kg})$.

Na Aninga, observou-se que o elemento que apresentou maior concentração foi também o Cálcio $(\mathrm{Ca}=31538,72 \mathrm{mg} / \mathrm{Kg})$ e o de menor concentração o Cobre $(\mathrm{Cu}=6,81 \mathrm{mg} / \mathrm{Kg})$. Vale lembrar, que nos gafanhotos, também, o Cálcio foi o elemento de maior concentração e o de menor o Cobre, semelhante à 
sua planta hospedeira. É importante destacar que a concentração de Cálcio em $T$. surinama em relação a planta é bem pequena.

O elemento Cobre, no gafanhoto apresentou maior concentração nos machos ( $\mathrm{Cu}=121,75 \mathrm{mg} / \mathrm{Kg}$ ) seguido dos imaturos (ninfas) ( $\mathrm{Cu}=48,44 \mathrm{mg} / \mathrm{Kg}$ ) e depois das fêmeas ( $\mathrm{Cu}=43,26 \mathrm{mg} / \mathrm{Kg}$ ).

A concentração do Zinco no gafanhoto não teve uma grande variação em relação à sua planta hospedeira. A maior concentração de Zinco foi encontrada nas fêmeas ( $Z n=280,11 \mathrm{mg} / \mathrm{Kg}$ ) seguido dos imaturos (ninfas) $(\mathrm{Zn}=226,71 \mathrm{mg} / \mathrm{Kg}$ ) e depois dos machos que tiveram pouca diferença em relação as ninfas ( $\mathrm{Zn}=225,02 \mathrm{mg} / \mathrm{Kg}$ ). Na Aninga a concentração desse mineral foi de 255,9 mg/Kg (Tabela 1).

Na composição mineral de $T$. surinama, o Manganês teve a maior concentração nos imaturos ( $\mathrm{Mn}=$ $135,07 \mathrm{mg} / \mathrm{Kg}$ ) seguido das fêmeas ( $\mathrm{Mn}=76,38 \mathrm{mg} / \mathrm{Kg}$ ) e depois pelos machos ( $\mathrm{Mn}=44,43 \mathrm{mg} / \mathrm{Kg}$ ). Essas concentrações são bem pequenas em relação à da sua planta hospedeira $M$. linifera $(\mathrm{Mn}=3612,23 \mathrm{mg} / \mathrm{Kg})$.

Com relação à concentração dos elementos minerais no gafanhoto $T$. surinama, pode-se admitir a existência de certo padrão entre os estágios, com exceção nas fêmeas e ninfas, referente ao Ferro, e aos machos quanto ao Cobre (Figura 3). Isso é percebido em decorrência da tendência para a diminuição da concentração, de tais elementos nos gafanhotos, que de forma contrária apresentaram concentrações com valores elevados. Esse resultado parece indicar que nos gafanhotos, existe uma forma diferente de assimilação/absorção desses minerais, em relação aos estágios de desenvolvimento desses insetos.

Considerando o possível padrão existente entre os elementos minerais contidos nos estágios de desenvolvimento de $T$. surinama, foi realizada uma análise do coeficiente de correlação entre os elementos químicos encontrados na Aninga e os ocorrentes nos gafanhotos, considerando as médias totais das amostras. Essa análise verificou a relação existente entre tais elementos em ambas as espécies. Os resultados dessa análise indicaram haver relação entre os componentes minerais da planta hospedeira e dos gafanhotos, visto que o coeficiente de correlação ( $r$ ) apresentou valores correspondentes a correlações, muito forte, forte e moderada (BRAGA, 2008) (Tabela 2). Esse resultado, também fortalece a ideia de que os elementos minerais existentes nos gafanhotos são provenientes da Aninga, da qual eles se alimentam.

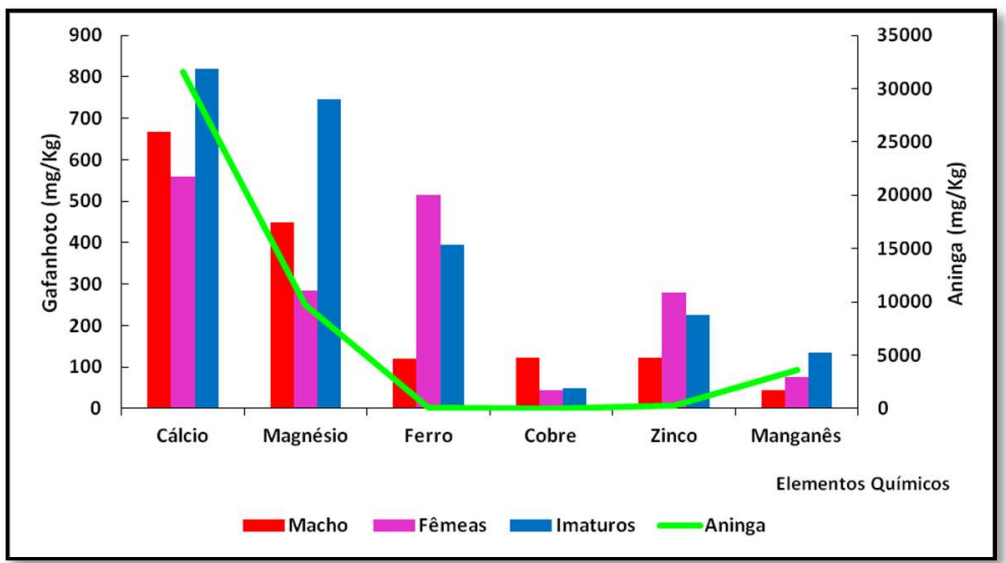

Figura 3: Concentração dos elementos minerais em T. surinama, por estágios de desenvolvimento do gafanhoto, em relação a sua planta hospedeira $M$. linifera. 
Tabela 2: Análise de correlação entre os valores de concentração de todos os elementos minerais analisados no gafanhoto $T$. surinama e sua planta hospedeira M. linifera.

\begin{tabular}{lll}
\hline Tratamento (Gafanhoto x Planta) & Valor do Coeficiente de Correlação ( $r$ ) & Correlação \\
\hline Fêmeas x Aninga & 0.5656 & Moderada \\
Ninfas x Aninga & 0.7961 & Forte \\
Média Total dos Gafanhotos x Aninga & 0.8293 & Forte \\
\hline
\end{tabular}

\section{Metais Pesados}

Neste estudo foram realizadas análises em T. surinama, para a determinação de dois metais pesados, em decorrência do fato de que a localidade onde foram feitas às coletas, tratar-se de uma região urbanizada que pode ter a incidência de algum metal pesado, oriundo de efluentes urbanos ou industriais, razão pela qual foi admitida a necessidade de verificar se havia o acúmulo de Cádmio e Chumbo no gafanhoto $T$. surinama.

Os resultados dessa análise mostram que a maior concentração de Cádmio foi encontrada nos imaturos $(C d=0,0082 \mathrm{mg} / \mathrm{Kg})$ seguido dos machos $(C d=0,0037 \mathrm{mg} / \mathrm{Kg})$ e nas fêmeas essa análise deu negativo, indicando não existir presença de Cádmio nas fêmeas do gafanhoto. A quantidade de Cádmio encontrada nos machos e imaturos é considerada baixíssima para ocasionar a contaminação do gafanhoto por esse elemento químico. Quanto à análise destinada ao Chumbo todas as três amostras para machos, fêmeas e imaturos deram negativo, indicando não haver presença de Chumbo na composição mineral de $T$. surinama.

\section{DISCUSSÃO}

Considerando que o cálcio e o magnésio foram os elementos de maior concentração em $T$. surinama, destaca-se que esse resultado é importante, pois o Magnésio é um mineral indispensável para realização de mais de 300 reações metabólicas, desempenhando papel fundamental no metabolismo dos animais (SEVERO et al., 2015). Carvalho (1999) evidenciou que uma das reações realizadas pelo Magnésio, refere-se ao auxílio da absorção do Cálcio nos organismos vivos. Dessa forma, geralmente em uma amostra onde se encontra o elemento Cálcio, consequentemente será encontrado o Magnésio, pois são elementos químicos que se complementam e, por isso, são encontrados juntos, apresentando concentrações equivalentes. Tal resultado pode ser corroborado com os obtidos na análise de elementos minerais da Aninga, a planta hospedeira de $T$. surinama. Em tal análise, que utilizou o mesmo protocolo empregado para este estudo no gafanhoto, tanto o Cálcio quanto o Magnésio estiveram presentes e evidenciaram concentrações muito elevadas (Tabela 1).

Quanto a maior concentração de Ferro em T. surinama, em comparação aos valores desse metal na Aninga, que indica haver mais Ferro nos gafanhotos do que na sua planta hospedeira ( $\mathrm{Fe}=58,82 \mathrm{mg} / \mathrm{Kg}$ ), da qual se alimenta, isso se deve, possivelmente, a condição acumulativa de metais no exoesqueleto dos insetos em geral, o que pode variar de acordo com as pressões ambientais e a alimentação (BROTTO et al., 2014).

Com relação ao fato da concentração de Ferro ter sido menor na planta, destaca-se que os valores dos metais em M. linifera, utilizados neste trabalho, são oriundos do estudo de Amarante et al. (2009), cuja as plantas de Aninga foram procedentes da margem direita do Rio Guamá, enquanto que o local de coleta 
dos gafanhotos e das folhas de Aninga, que serviram de alimento aos mesmos (neste estudo), antes da análise química, foram coletados numa área de baixio no Campus da UFRA, a qual apenas sofre influência das mares do rio Guamá. Deve-se considerar, ainda, que este rio banha a cidade de Belém, capital do estado do Pará, o qual recebe os efluentes do meio urbano deste município e, que por isso, pode conter em suas águas um maior aporte de Ferro.

É também importante considerar a relação Manganês/Ferro nos tecidos de plantas, pois a toxidez do Manganês está associada à deficiência de Ferro (AMARANTE et al., 2009), o que sugere que amostras cujos valores de Manganês estiverem elevados, consequentemente os de ferro estarão baixos, o que poderia justificar os resultados obtidos nas análises da Aninga, abordados neste estudo, visto que os valores de Manganês evidenciados são completamente inversos (Tabela 1).

É importante considerar que o Ferro é um elemento fundamental nos vertebrados, pois ele faz o transporte do oxigênio para as células por meio do sistema circulatório. Vasconcelos (2017), explica que o sistema circulatório dos insetos não desempenha uma função vital no transporte de oxigênio. Entretanto, é sabido que nos invertebrados e vertebrados, o Ferro além de ser um elemento essencial para os processos de oxirredução, quando acumulado, pode causar implicações nocivas para os insetos (NICHOL et al., 2002). Essas implicações podem ser causadas por uma grande quantidade de Ferro no organismo, mesmo que seja além do que os organismos possam metabolizar. Entretanto, o Ferro é um mineral vital para a homeostase celular e a sua habilidade em aceitar e doar elétrons, o torna imprescindível para diversas reações biológicas (GROTTO, 2010), inclusive em T. surinama, sendo, portanto, muito importante no transporte de ions nos diversos sistemas metabólicos do gafanhoto.

Com a escassez de literatura sobre a alta concentração de Ferro nos insetos, fica difícil tecer considerações sobre as causas da elevada concentração desse elemento, observada nas fêmeas do $T$. surinama, e qualquer inferência sobre tal resultado, poderia ser considerada mera especulação. Contudo, esse resultado impulsiona a realização de pesquisas futuras, que possam explicar os dados obtidos neste estudo.

O Cobre como os outros elementos químicos é indispensável nos seres vivos, e igualmente ao Ferro, teve maior concentração nos gafanhotos do que na Aninga $(C u=6,81 \mathrm{mg} / \mathrm{Kg})$, o que pode ser proveniente do acumulo desse metal no exoesqueleto do gafanhoto, por exemplo. Vale ressaltar, que a existência deste microelemento no organismo animal, é importante, pois desempenha várias funções vitais, como participar da transferência de elétrons nas atividades enzimáticas de oxirredução. Fisiologicamente é indispensável para a vida, como também em diversas reações bioquímicas (DÍAZ et al., 2015). Deve-se destacar que o citocromo oxidase, uma metaloenzima que contém dois íons de cobre, é determinante na transferência de elétrons para o oxigênio (redução de O2 à água), etapa fundamental na respiração celular (LEHNINGER, 1995) dos animais.

Referindo-se ao elemento Zinco, destaca-se que este metal tem uma melhor assimilação, nos organismos vivos, quando na presença do Magnésio (McDOWELL, 1992). Cruz et al. (2011) descreveram a importância do Zinco no século atual, especialmente nos últimos anos, pois este mineral é essencial em 
funções metabólicas de plantas e animais. A baixa quantidade de Zinco no organismo animal pode afetar a taxa de crescimento, influindo negativamente no desenvolvimento desses organismos. Este elemento também desenvolve uma função essencial na defesa do organismo, fortalecendo as células do sistema imunológico, tornando-o menos vulnerável a infecções (ISHIKAWA et al., 2008; CRUZ et al., 2011).

Com relação ao Manganês que no gafanhoto teve baixa concentração em relação à da sua planta hospedeira M. linifera, Ruppenthal (2013) destaca que esse minério se encontra amplamente distribuído no solo, sedimentos, rochas, água e materiais biológicos, sendo um elemento essencial para o desenvolvimento das plantas, razão que pode justificar o grande aporte deste, pela macrófita. Entretanto, embora o Manganês atue nos processos vitais dos animais, este elemento parece não possuir propriedade acumulativa no organismo do gafanhoto, o que indica ser contrário quanto ao Ferro e o Cobre. Pode-se ainda inferir, que diante desse resultado parece que $T$. surinama possui algum mecanismo metabólico que permite a excreção do Manganês.

A indicação da existência de uma forma diferente de assimilação/absorção dos minerais, em relação aos estágios de desenvolvimento do gafanhoto estudado, destaca-se que tal afirmação pode ser aceitável, principalmente quanto às fêmeas e imaturos, pois as fêmeas devido a condição de geração de ovos, processo de oviposição e o porte e peso avantajado (fêmeas de $T$. surinama são maiores e mais pesadas que os

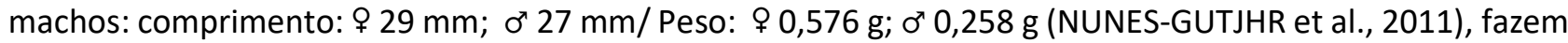
a ingestão de maior quantidade de Aninga e consequentemente dos minerais contidos nas folhas dessa macrófita aquática. Essa afirmação, também pode ser aceitável para as ninfas do gafanhoto, visto que se tratando de imaturos, estes necessitam comer mais para atingir seu completo desenvolvimento. Esse resultado, já foi observado para o gafanhoto semiaquático Cornops frenatum frenatum (Acrididae, Leptysmine) quando num estudo de consumo alimentar, as ninfas apresentaram maior consumo foliar em relação aos adultos e as fêmeas em relação aos machos (RESQUE JÚNIOR, 2011).

O fato da concentração dos minerais ser diferente em cada estágio do gafanhoto, sendo maior nas fêmeas e nos imaturos de $T$. surinama e em relação a Aninga, é importante destacar que estatisticamente houve forte correlação $(r=08293)$ entre a concentração média de minerais no gafanhoto e sua planta hospedeira.

Vale ressaltar, que em certas condições os elementos metais podem ser prejudiciais, e até mesmo alcançar altos níveis de toxidade para os seres vivos (VAITSMAN et al., 2006). Isso pode ocorrer quando existe muito vestígio de metais disponíveis, que não irão desempenhar uma função vital ou essencial dentro do organismo (SPIRO et al., 2009) e principalmente se estes forem acumulativos. Em alguns casos os insetos que se alimentam de certos compostos tóxicos, desenvolvem estratégias fisiológicas para sobreviver à intoxicação. Uma das alternativas para tal situação refere-se ao fato de que tais insetos podem induzir a produção de enzimas de detoxicação, como por exemplo, o citocromo P450 (proteínas transportadoras de elétrons e catalizadoras) que se encontra localizada no intestino médio dos insetos (BRATTSTEN et al., 1977). Frente a essas afirmações, pode-se admitir que os metais em maior concentração em $T$. surinama (elementos acumulados em seu corpo), podem ter a toxidade diminuida, por meio de mecanismos metabólicos como o 
do citocromo P450.

Portanto, nem todo alimento tóxico que os insetos possam ingerir será prejudicial a ele ou até mesmo, para outro animal que dele possa se alimentar (na predação), pois, como mencionado anteriormente, às vezes a grande concentração de um elemento acumulativo, pode corresponder a um fator para desencadear a produção de enzimas necessárias para desintoxicar seu organismo, ou até mesmo, para ajudar na metabolização de outros elementos.

Segundo a Agência Nacional de Vigilância Sanitária (ANVISA, 2005), para humanos o nível tolerável diário, por exemplo, de manganês para um grupo populacional por faixa etária é o seguinte: de 1 a 3 anos é de 1,2 mg; de 4 a 10 anos é de 1,5 mg; para adultos é de 2,3 mg; para gestantes é de 2,0 mg e para lactantes é de 2,6 mg. Considerando estes valores de tolerância, é perfeitamente justificável a designação "planta tóxica" para a Aninga, pois os valores de concentração do Manganês, contido na mesma, é muito acima do nível diário tolerável.

Embora o gafanhoto $T$. surinama, se alimente da folha de Aninga, que é uma planta tóxica devido seu alto nível de Manganês, em decorrência dos resultados obtidos neste estudo, não será considerado tóxico para os seres humanos (Manganês: Machos=0,4 mg; Fêmeas=0,07 mg e Imaturos=0,13 mg), caso pessoas venham se alimentar deste inseto, o que é comum em muitas culturas do mundo que praticam a entomofagia. Atualmente, é sabido que possivelmente há mais de 1.000 espécies de insetos que estão sendo ou já serviram de alimento para humanos em algum lugar do mundo (GULLAN et al., 2012), em decorrência do hábito alimentar, necessidade ou devido a comportamentos culturais.

Pelo exposto, pode-se afirmar que para os possíveis predadores (consumidores) de $T$. surinama, em uma cadeia alimentar, tais como pequenos mamíferos, roedores, aves, répteis, anfíbios e aracnídeos, esse gafanhoto não será tóxico, visto que o nível de Manganês existente em seu organismo está em pequena quantidade, embora esse gafanhoto apresente coloração aposemática, que indica alerta aos seus predadores (Figura 4). Portanto, o padrão cromático (aposematismo), observado em T. surinama, pode ser considerado uma estratégia de sobrevivência dessa espécie de gafanhoto, decorrente do processo de co-evolução desse ortóptero com sua macrófita hospedeira $M$. linefera.

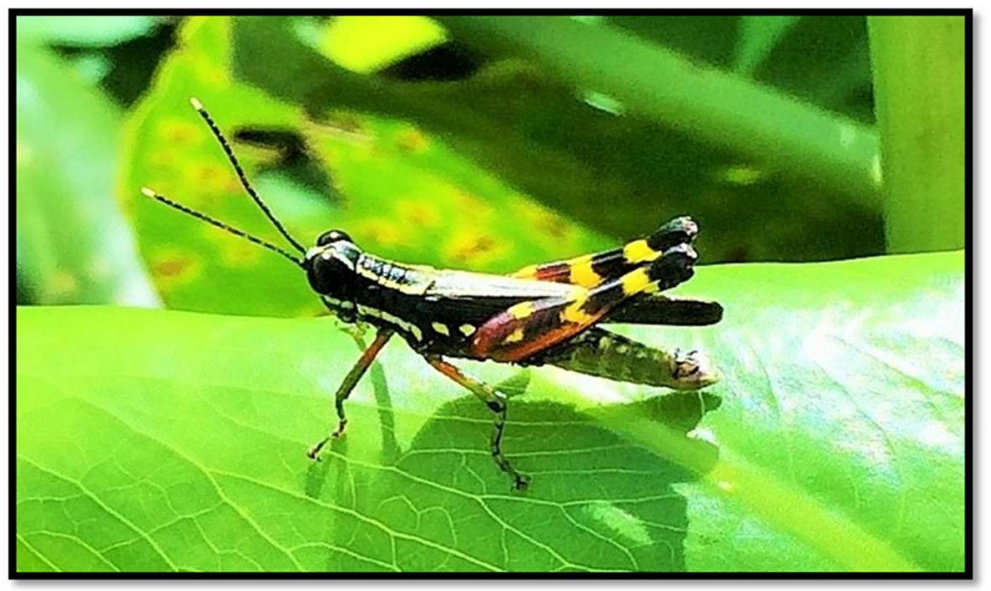

Figura 4: Aspecto de Tetrataenia surinama, evidenciando sua coloração aposemática. 


\section{Metais pesados}

É sabido que os metais pesados são aqueles que apresentam a densidade maior que $5,0 \mathrm{mg} / \mathrm{cm}^{3}$ (ATKINS et al., 1992), sendo bioacumulativos e muito reativos, o que dificulta e impedem que os organismos vivos eliminem ou possam expelí-los de uma forma eficaz e rápida (COLACIOPPO, 2001). Quanto a esses elementos, Colacioppo (2001) relata que os metais como o Chumbo $(\mathrm{Pb})$ e o Cádmio $(\mathrm{Cd})$ não se encontram espontaneamente nos seres vivos e não possuem funções bioquímicas e nem nutricionais em organismos como planta e animais, indicando que esses metais somente podem ser absorvidos por plantas e animais por meio de ingestão e assimilação,

Neste estudo, os valores de concentração de Cadmio Chumbo em $T$. surinama foram ínfimos e inexistentes, indicando que o gafanhoto não representa um difusor dos metais Cádmio e Chumbo em uma cadeia alimentar ou trófica, embora sejam necessários estudos complementares para gerar informações mais completas.

Os resultados obtidos para o Cádmio e Chumbo, neste estudo, constitui uma importante informação de cunho ambiental, pois é um indicativo de que tanto a água da área de estudo, onde foram coletados os gafanhotos, quanto a Aninga que lá se encontra, praticamente não tem incidência de Cádmio e Chumbo, indicando que esses metais se encontram em pouca quantidade no local onde foram coletados os gafanhotos.

\section{CONCLUSÕES}

Este estudo proporcionou a identificação da presença dos elementos minerais, Cálcio, Magnésio, Ferro, Zinco, Cobre e Manganês no gafanhoto $T$. surinama e entre os principais elementos minerais evidenciados nesse inseto, o Cálcio e o Magnésio foram os que ocorreram em maior concentração, pois, são elementos que são encontrados juntos na natureza.

Embora $T$. surinama se alimente de $M$. linifera, que possui grande concentração de manganês, este gafanhoto evidenciou baixa concentração de desse mineral, indicando que o mesmo não retém tal elemento, o que indica que $T$. surinama não possui características tóxicas, para os seus predadores, apesar de ser um inseto que possui aposematismo (cores de advertência). Tal coloração intensa pode ser considerada um mecanismo de defesa do gafanhoto, contra seus predadores, resultado de processos coevolutivos de $T$. surinama e de sua planta hospedeira M. linifera.

A análise mineral de $T$. surinama evidenciou uma boa quantidade de Ferro, Cálcio e Magnésio, sugerindo que esse gafanhoto pode ser bem nutritivo para quem se alimentar do mesmo, não descartando a possibilidade de incluí-lo como item em estudos de entomofagia para humanos.

A análise química realizada para pesquisa de metais pesados não identificou a presença de chumbo no gafanhoto $T$. surinama, porém foi constatada nos machos e nos imaturos a presença de uma pequena quantidade de Cádmio. Essa informação é importante, pois indica uma boa condição ambiental, quanto a esses metais pesados, no local onde o gafanhoto foi coletado, visto que se trata de uma localidade no 
perímetro urbano, onde os despejos de esgotos, principalmente de fábricas, podem lançar tais elementos no meio aquático, onde se encontram os aningais e consequentemente o gafanhoto $T$. surinama.

\section{REFERÊNCIAS}

ANVISA. Agência Nacional de Vigilância Sanitária. Resolução de Diretoria Colegiada - RDC no 269, de 22 de setembro de 2005. Regulamento Técnico Sobre a Ingestão Diária Recomendada (IDR) de Proteína, Vitaminas e Minerais. ANVISA, 2005.

AMARANTE, C. B.; MÜLLER, R. C. S.; DANTAS, K. G. F.; ALVES, C. N. MÜLLER A. H.; PALHETA, D. C.. Composição química e valor nutricional para grandes herbívoros das folhas e frutos de aninga (Montrichardia linifera,Araceae). Acta Amazônica, v.40, n.4, p.729-736, 2010.

AMARANTE, C. B.; SILVA, J. C. F.; SOLANO, F. A. R.; NASCIMENTO, L. D.; MORAES, L. G.; SILVA, G. F.; UNO, W. S. Estudo Espectrométrico Das Folhas Da Aninga (Montrichardia Linefera) Coletadas à Margem do Rio Guamá no Campus Da UFPA, Belém-PA. Uma Contribuição ao Estudo Químico da Família Araceae. Revista Científica da UFPA, v.7, n.1, p.1-15, 2009.

AMARANTE, C. B.; SILVA, J. C. F.; MÜLLER, R. C. S.; MÜLLER, A. H.. Avaliação da composição mineral do chá da folha senescente de Montrichardia linifera (Arruda) schott (Araceae) por espectrometria de absorção atômica com chama (FAAS). Quím. Nova, v.34, n.3, p.419-423, 2011.

ATKINS, P.; JONES, L.. Chemistry: Molecules, matter, and change. 3 ed. New York: W. H. Freeman and company, 1992.

BRAGA, C. E. S.. Aspectos fenológicos e consumo alimentar de Cornops Aquaticum (Bruner, 1906) (Orthoptera: Acrididae: Leptysminae) associado a eichhornia crassipes (mart.) Solms (pontederiaceae) em dois lagos da Amazônia Central, Brasil. Dissertação (Mestrado em Entomologia) Instituto Nacional de Pesquisas da Amazônia, Manaus, 2008.

BRATTSTEN, L. B.; WILKISON, C. F.; EISNER, T.. Herbivore plant interractions mixed function oxidases and secondary plant. Science, New York, v.196, p.1394-1349, 1977.

BROTTO, D. F.; FIRMINO, E. L. B.. Insetos como bioindicadores e acumuladores de poluição por metais pesados. In: ENEPEX - ENCONTRO DE ENSINO PESQUISA E EXTENSÃO, 6; ENEPE UFGD, 8; EPEX UEMS, 5. Anais. Dourados, 2014.

CARVALHO, P. R. C.. Medicina Ortomolecular: um guia completo sobre os nutrientes e suas propriedades terapêuticas. 4 ed. Rio de Janeiro: Nova Era, 1999.

COLACIOPPO, S.. Higiene e toxicologia ocupacional: Metais Pesados. In: JORNADA DE TOXICOLOGIA DA SOCIEDADE PAULISTA DE MEDICINA DO TRABALHO E DO DEPARTAMENTO DE MEDICINA DO TRABALHO DA ASSOCIAÇÃO PAULISTA DE MEDICINA UNIVERSIDADE DE SÃO PAULO, 1. Anais. 2001.

COSTA, R. A. C. V.; VIEIRA, M. F.. Determinação da Especificidade Alimentar de Tetrataenia surinama (Orthoptera: Acrididae: Leptysminae em Condições Naturais de Laboratório). In: JORNADA DE INICIAÇÃO CIENTÍFICA DO INPA, 7. Anais. Manaus, 1998.

COSTA NETO, E. M.; JRESENDE, J. J.. A percepção de animais como "insetos" e sua utilização como recursos medicinais na cidade de Feira de Santana, Estado da Bahia, Brasil. Acta Scientiarum. Biological Sciences, Maringá, v.26, n.2, p.143149, 2004.

CRUZ, J. B. F.; SOARES, H. F.. Uma revisão sobre o zinco. Ensaios e Ciência: Ciências Biológicas, Agrárias e da Saúde, v.15, n.1, p.207-222, 2011.

DÍAZ, T. G.; TEODORO, A. L.; ROJAS, I. C. O.; CHITIVA, A. F. P.; GUZMAN, J. A. P.. Metabolismo do cobre na nutrição animal: Revisão. PubVet. Maringá. v.9, n.5, p.279-286, 2015.

GROTTO, H. Z.. Fisiologia e metabolismo do ferro. Rev. Bras. Hematol. Hemoter, São Paulo, v.32, n.2, p.8-17. 2010.

GULLAN, P. J.; CRANSTON, P. S.. Os insetos: um resumo de entomologia. 4 ed. São Paulo: Roca, 2012.

ISHIKAWA, Y.; KUDO, H.; SUZUKI, S.; NEMOTO, N.; SASSA, S.; SAKAMOTO, S.. Down regulation by a low-zinc diet in gene espression of rat prostatic thymidylate synthase and thymidine kinase. Nutrition \& Metabolism, v.5, n.12, p.1-5. 2008.

JELASKA, L. S.; BLANUŠA, M.; DURBEŠIĆ, P.; JELASKA, S. D. Heavy metal concentrations in ground beetles, leaf letter, and soil of a forest ecosystem. Ecotoxicology and Environmental Safety, v.66, n.1, p.74-81, 2007.

LEHNINGER, A. L.. Princípios de bioquímica. São Paulo: Savier, 1995.

McDOWELL, L. R.. Minerals in animal and human nutrition San Diego: Academic Press Inc., 1992.

NICHOL, H., LAW, J. H.; WINZERLING, J. J.. Iron metabolism in insects. Ann. Rev. Entomol., v.47, n.1, p.535-552, 2002.

NUNES, A. L.. Estudo sobre o ciclo de vida e fenologia de Stenacris fissicauda fissicauda (Bruner, 1908) (OrthopteraAcrididae) em um lago de várzea da Amazônia Central, Brasil, Manaus. Dissertação (Mestrado em Entomologia) Instituto Nacional de Pesquisas da Amazônia, Manaus, 1989.

NUNES-GUTJAHR, A. L.; BRAGA, C. E. S.. Gafanhotos da Flona Caxiuanã: Guia Prático. Belém: Museu Paraense Emílio Goeldi, 2011.

PACIULLO, D. S. C.; DERESZ, F.; LOPES, F. C. F.; AROEIRA, L. J. M.; MORENZ, M. J. F.; VERNEQUE, R. S.. Disponibilidade de matéria seca, composição química e consumo de forragem em pastagem de capim-elefante nas estações do ano. Arq. Bras. Med. Vet. Zootec., v.60, n.4, p.904-910, 2008.

PILLA, M. A. C.; AMOROZO, M. C. M.; FURLAN, A.. Obtenção 
e uso das plantas medicinais no distrito de Martim Francisco, Município de Mogi-Mirim, SP, Brasil. Acta Bot. Bras., v.20, n.4, p.789-802, 2006.

RESQUE JÚNIOR, B. T. B.. Estudo sobre o Consumo Alimentar de Cornops frenatum frenatum (MARSCHALL, 1836) (Orthoptera, Acrididae, Leptysminae). Monográfica (Bacharelado em Ciências Naturais, Biologia) - Universidade do Estado do Pará, Belém, 2011.

RAFAEL, A. L.; MELO, G. A. R.; CARVALHO, C. J. B.; CASARI, S. A.; CONSTANTINO, R.. Insetos do Brasil: Diversidade e Taxonomia. Ribeirão Preto: Holos, 2012.

RODRIGUES, L.; MEIRELLES, P. R. L.; GONÇALVES, H. C.; MARTINS, M. F.; MARQUES, R. O.; ARRUDA, G. M. M. F.. Disponibilidade e composição química do capim-tanzânia, pastejado por caprinos. Veterinária e Zootecnia, v.17, n.4, p.585-595, 2010.

RUIVO, J. S. P.. Fitocosmética: aplicação de extratos vegetais em Cosmética e Dermatologia. Dissertação (Mestrado em Ciência Farmacêuticas) - Universidade Fernando Pessoa, Porto, 2012.

RUPPENTHAL, J. E.. Toxicologia. Santa Maria: Universidade Federal de Santa Maria, 2013.

SEVERO, J. S.; MORAIS, J. B. S.; FREITA, T. E. C.; CRUZ, K. J. C.; OLIVEIRA, A. R. S.; POLTRONIERI, F.; MARREIRO, D. N..

Aspectos metabólicos e nutricionais do magnésio. Nutr. Clín. Diet. Hosp., v.35, n.2, p.67-74, 2015.

SPIRO, T. G.; STIGLIANI, W. M.. Química ambiental. 2 ed. São Paulo: Pearson pretice Hall, 2009.

VAITSMAN, E. P.; VAITSMAN, D. S.. Química e meio ambiente: ensino contextualizado. Rio de Janteiro: Interciência, 2006.

VASCONCELOS, S.. Zoologia dos invertebrados superiores. Lavras: UFLA, 2017.

A CBPC - Companhia Brasileira de Produção Científica (CNPJ: 11.221.422/0001-03) detém os direitos materiais desta publicação. Os direitos referem-se à publicação do trabalho em qualquer parte do mundo, incluindo os direitos às renovações, expansões e disseminações da contribuição, bem como outros direitos subsidiários. Todos os trabalhos publicados eletronicamente poderão posteriormente ser publicados em coletâneas impressas sob coordenação da Sustenere Publishing, da Companhia Brasileira de Produção Científica e seus parceiros autorizados. Os (as) autores (as) preservam os direitos autorais, mas não têm permissão para a publicação da contribuição em outro meio, impresso ou digital, em português ou em tradução. 